\title{
Frequency Spectra of Atmospheric Motions in the Vicinity of a Mountain Barrier
}

\author{
Julia Nogues Paegle and Jan Paegle \\ Department of Meteorology, University of Utah, Salt Lake City 84112
}

(Manuscript received 23 July 1974, in revised form 5 November 1975)

\begin{abstract}
Frequency spectra of heights and geostrophic vorticities are computed for several points over the western continental United States and eastern Pacific. These spectra exhibit horizontal variations which appear to be, at least partly, attributable to the underlying topography. This conclusion is supported by a highly simplified, barotropic, mountain-flow model.
\end{abstract}

\section{Introduction}

Numerous studies have pointed out the effects that the Rocky Mountains may have on the synoptic and planetary scale flow. McClain (1960), Kao et al. (1974), Paegle and Paegle (1975) and others have analyzed synoptic-scale data in the vicinity of the Rockies and emphasized certain apparent terrain influences. Theoretical works by Queney (1948), Charney and Eliassen (1949), Bolin (1950) and others have considered steady mean flows. Numerical experiments of Mintz (1965), Kasahara and Washington (1971), and Manabe and Terpstra (1974), among others, have tried to isolate the effect of orography for stationary and transient disturbances.

In the present investigation quantitative estimates of the Rocky Mountain effect upon the synoptic-scale circulation are presented in the form of height and geostrophic vorticity frequency spectra (Section 2). The principal result is that the variance contained in the long-period oscillations of these spectra is about twice as great upwind of the mountains as it is downwind, whereas shorter period fluctuations exhibit the opposite distribution. It might consequently be hypothesized that this is a region where energy transformation from long to short period oscillations occurs, and, furthermore, that the mountains have a determining influence in this transformation. Although such a conclusion would not be inconsistent with some of the other investigations that are cited above, the precise cause-effect relationship is not immediately apparent from the simulations. The analytical treatments have been steady-state, and the numerical investigations include many influences in addition to topography.

Lee wave development is both a baroclinic and barotropic phenomenon and there are various surface inhomogeneities in addition to the mountains. We in- vestigate the simplest model which might have relevance in Section 3 in order to address the cause-effect question. A time-dependent, barotropic, potentialvorticity-conserving flow is simulated over a latitudinally uniform step function model of the Rocky Mountains. The conclusion is that slow oscillations of the zonal flow, that are characteristic of index cycle oscillations, can spread significant energy into higher synoptic-scale frequencies as a consequence of terrain effects in simple barotropic flows.

\section{Computational methods and spectral results}

The data consist of grid-point heights at 1200 and 2400 GMT extracted from the National Meteorological Center (NMC) analyses for three winter seasons (December, January and February, 1965-67). The results are presented for the three winter averages and for three frequency bands which are of interest in our analysis. Missing points, which accounted for less than $5 \%$ of the total sample, were linearly interpolated. Geostrophic vorticities were computed using centered finite differences on a sub-set of the NMC octagonal grid.

The spectral results were obtained using a cosine taper on the original time series and then combined to display the contributions to the time variance for three time scales: longer than 11 days (scale 1), from 4 to 11 days (scale 2), and from 2 to 4 days (scale 3). These results are associated with 60,90 , and 120 degrees of freedom for scales 1 through 3 , respectively, indicating a high level of statistical reliability.

The $700 \mathrm{mb}$ vorticity variances are presented in Fig. 1. Two well-defined maxima may be discerned, one over the Gulf of Alaska for scale 1, and another over and to the east of the Rocky Mountains for scales 2 and 3 . The former maximum is related to a region of 
(A)
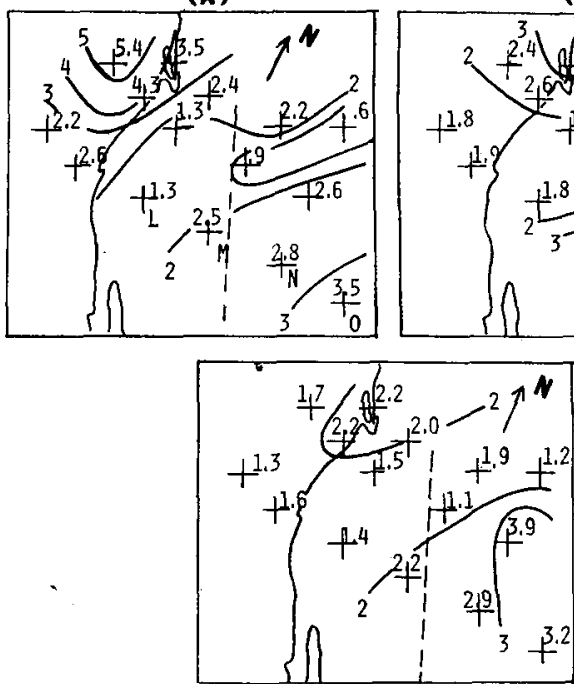

(c)

FIG. 1. Contributions to the time variance of $700 \mathrm{mb}$ absolute geostrophic vorticities $\left(10^{-10} \mathrm{~s}^{-2}\right)$ from scales 1,2 and 3 (A, B and $\mathrm{C}$, respectively). The dashed vertical line indicates the approximate location of the eastern slope of the Rocky Mountains.

strong variability associated with the semi-permanent low pressure center which prevails in the Gulf of Alaska (Paegle and Keirulff, 1974). The higher frequency maxima over and to the east of the Rocky Mountains might be, at least partly, consequences of the mountains. The spectra of heights around $40^{\circ} \mathrm{N}$ show a similar effect (Fig. 2). The Rocky Mountains present their broadest cross section to the ambient westerly flow at about $40^{\circ} \mathrm{N}$, and here the mountain effect on the kinematics should be most apparent. It is noteworthy that the strongest variances for long-period oscillations are about twice as strong upwind of the

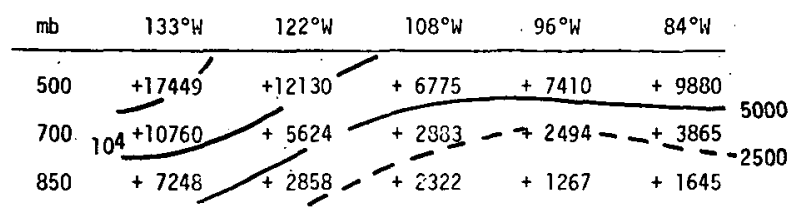

(a)

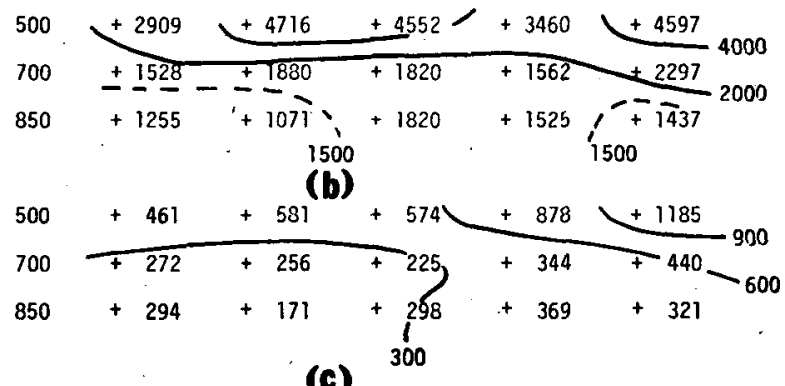

(c)

Fig. 2. Contributions to the time variance of heights $\left(\mathrm{m}^{2}\right)$ at about $40^{\circ} \mathrm{N}$ for scales $1-3$. mountains as they are downwind, while the highfrequency variance displays the reverse distribution.

A similar variation was found in Paegle and Paegle (1975) in the frequency spectra of quasi-geostrophic motions, which presented markedly different characteristics across the Rocky Mountains. The higher frequency components to the east of the mountains in the kinematic fields may be interpreted qualitatively as a result of the developments which are characteristic of this region. In unpublished case studies using numerical quasi-geostrophic flow simulations (Paegle and Kousky, 1972), we have found increases in baroclinic wave activity and consequent development in this region because of the Rockies. It is difficult to control the upwind flow oscillation in such simulations and they are relatively uneconomical for extended tests of the basic physics. In fact, a much simpler approach is apparently sufficient to explain the spectral shifts and we, therefore, consider an analytic model of the influence of the mountains on a barotropic flow.

\section{Mathematical orographic model}

Bolin (1950) has pointed out that the dominating flow deformation caused by the Rocky Mountains results from the fact that the westerlies are forced to flow over these ranges rather than around. Therefore, we have followed the approach discussed by Batchelor (1967) and considered an infinite ridge extending in the north-south direction. Observational studies (Kao and Wendell, 1970; Kao et al., 1970) have shown that the main contribution to the variance of the zonal wind comes from very long periods. As a first approximation, we allow the mean zonal flow to be a slowly varying function of time, simulating an index cycle, and study how this oscillation would affect the frequency spectra of vorticity and streamfunction.

Under equilibrium conditions the wavelength of the disturbances induced downstream from a mountain range is a function of the strength of the zonal flow. As the zonal flow slowly oscillates in time, a location downstream from the mountains finds itself alternately in a trough or ridge position, with a period of oscillation which may be considerably shorter at this location than the period of the zonal flow oscillation. The purpose of the following analysis is to check this effect quantitatively within the framework of barotropic potentialvorticity-conserving dynamics.

\section{a. Flow over and downwind of a ridge}

We consider a basic zonal flow which varies very slowly in time, with a time scale $\tau=t /$ Ro, where Ro is a Rossby number assumed much less than 1 and $t$ is an advective time scale. For a first-order approximation, particle trajectories are essentially streamlines and we can apply Batchelor's (1967) analyses to obtain the flow field over the mountain profile (region A) given 


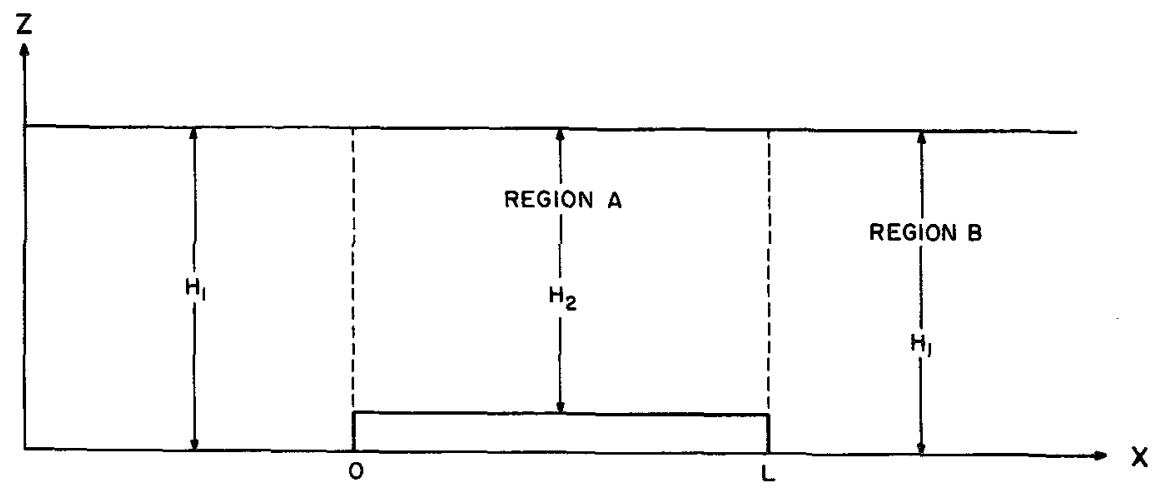

FIG. 3. Mountain model.

in Fig. 3. The other assumptions are the same as those used by Batchelor and need not be repeated here.

We can write

$$
\begin{array}{r}
\psi_{A}=-U_{A}(0)\{y+(1-c)[1+(B \text { Ro }) y][1-\cos (\alpha x)] / \\
(c B \text { Ro })\},
\end{array}
$$

where

$$
\begin{gathered}
\mathrm{R} 0=V /\left(f_{0} l\right) ; \quad U_{A}(0)=U_{A}(x=0) ; \\
c=H_{2} / H_{1} ; \quad \alpha=\left(\frac{B C}{U_{A}(0)}\right)^{2} ; \quad B=\frac{\beta_{0} l^{2}}{V} .
\end{gathered}
$$

In the above $f_{0}$ and $\beta_{0}$ are the Coriolis and beta parameters, respectively, at a typical latitude, $l$ and $V$ are typical length and velocity scales, $\psi$ is the streamfunction, and $x$ and $y$ are the zonal and meridional coordinates, respectively. All quantities are in nondimensional form. For large scales and middle latitudes $B \approx 1$.

To obtain the streamfunction for region $\mathrm{B}$ we use conservation of potential vorticity and mass continuity at $x=L$ which give the following differential equation and boundary conditions:

$$
\begin{gathered}
\operatorname{Ro}\left[\nabla 2 \psi_{B}+\delta^{2} \psi_{B}\right] \\
=[(c-1) / c][\cos (\alpha L)-1]-(B \mathrm{Ro}) y, \\
U_{B}(L)=U_{A}(0)[1+(c-1) \cos (\alpha L)], \\
V_{B}(L)=U_{A}(0)(c-1) \alpha \sin (\alpha L) \\
\times[1+(B \mathrm{Ro}) y] /(B \mathrm{Ro}),
\end{gathered}
$$

where $\delta=\alpha^{2} / c^{2}$.

The solution of (2) with (3) and (4) is given by

$$
\begin{aligned}
\psi_{B} & =-U_{A}(0)\left\{c y+(1-c)\left[(1+B \text { Ro } y) \cos \left(\frac{\alpha}{c}(x-L)\right)\right]\right. \\
& +\left(\frac{c-1}{2}\right)\left[\cos \left(\frac{\alpha}{c}-[x-(1+c) L]\right)-\left(\frac{c+1}{2}\right)\right. \\
& \left.\left.\times \cos \left(\frac{\alpha}{c}[x-(1-c) L]\right)+\cos (\alpha L)-1\right] /(B \text { Ro })\right\},
\end{aligned}
$$

and the vorticities in both regions are

$$
\begin{gathered}
\xi_{A}=-\left(\frac{1-c}{\operatorname{Ro}}\right)(1+B \operatorname{Ro} y) \cos (\alpha x) \\
\xi_{B}=\left(\frac{1-c}{c \text { Ro }}\right)(1+B \operatorname{Ro} y)\left[\cos \left(\frac{\alpha}{c}(x-L)\right)\right. \\
+\left(\frac{c-1}{2}\right) \cos \left(\frac{\alpha}{c}[x-(1+c) L]\right) \\
\left.-\left(\frac{c+1}{2}\right) \cos \left(\frac{\alpha}{c}[x-(1-c) L]\right)\right]
\end{gathered}
$$

where $\psi_{A}, \psi_{B}, \xi_{A}$ and $\xi_{B}$ are functions of the long-time scale $\tau$ through the dependence of the upstream flow $U=c U_{A}(0)$ on $\tau$.

The time dependence in (1), (5), (6) and (7) comes from three types of terms:

$$
U_{A}(0), U_{A}(0) \cos \frac{\phi}{\left(U_{A}(0)\right)^{\frac{1}{2}}}, \cos \frac{\phi}{\left(U_{A}(0)\right)^{\frac{1}{2}}} .
$$

Therefore, if we assume:

$$
U_{A}(0)=1+A \sin \omega \tau,
$$

it is of interest to compute the frequency spectra for

$$
\left.\begin{array}{l}
g_{1}(\tau)=(1+A \sin \omega \tau) \cos \frac{\phi}{(A \sin \omega \tau+1)^{\hat{\beta}}} \\
g_{2}(\tau)=\cos \frac{\phi}{(A \sin \omega \tau+1)^{\frac{1}{2}}}
\end{array}\right\} .
$$

This was done numerically for $0.1 \leqslant A \leqslant 0.9$ in increments of 0.1 and for $30^{\circ} \leqslant \phi \leqslant 360^{\circ}$ in increments of $30^{\circ}$ using a fast Fourier transform algorithm to obtain the spectra for 32 frequency bands. These values were chosen to cover the range of possible values. The higher resolution frequency spectra were combined into 6 main bands (Table 1) to summarize the results. The band $\Delta f_{0}$ defines the total variance of the function. 
TABLE 1. Frequency bands chosen to study the spectral characteristics of the analytic model.

Frequency band Contribution from

$\begin{array}{cc}\Delta f_{0} & \omega \leqslant \mathrm{f} \leqslant 32 \omega \\ \Delta f_{1} & f=\omega \\ \Delta f_{2} & 2 \omega \leqslant f \leqslant 4 \omega \\ \Delta f_{3} & 5 \omega \leqslant f \leqslant 8 \omega \\ \Delta f_{4} & 9 \omega \leqslant f \leqslant 16 \omega \\ \Delta f_{5} & 17 \omega \leqslant f \leqslant 32 \omega\end{array}$

Figs. 4 and 5 show the variance and fractional contribution of each frequency band to the total variance as a function of $A$ and $\phi$, for $g_{1}$ and $g_{2}$. As shown above, $g_{1}$ and $g_{2}$ appear in the definitions of the streamfunction and vorticity, respectively. The total variance of $g_{1}$ increases with increasing amplitude $A$, while a maximum value is found for $g_{2}$ at $A=0.6$. For both functions this maximum variability occurs at about $\phi=240^{\circ}$. Here we interpret $\phi$ as a measure of the downstream distance of the observation point.

For small $A$ most of the contribution of the frequency spectra appears in $\Delta f_{1}$, which corresponds to the forcing frequency, except for $\phi=180^{\circ}$ and $\phi=360^{\circ}$. For $g_{1}$ at $360^{\circ}$ and large $A$, and for $g_{2}$ at $180^{\circ}$ and $360^{\circ}$ for large and small $A$, the main contribution comes from $\Delta f_{2}$. While all other bands contribute less than $10 \%$ for $g_{1}$, contributions as high as 33 and $36 \%$ are found for $g_{2}$ from $\Delta f_{3}$ and $\Delta f_{4}$, respectively, for large $A$ and large $\phi$. Therefore, this model predicts larger contributions for $g_{2}$ than for $g_{1}$ at higher frequencies.
The strength of the mean flow oscillation and the location of the observation point are critical parameters in determining the characteristics of the frequency spectra in this model. For example, as the point $x=0$ is approached from the east, (1) and (6) show that the streamfunction time series at this point oscillates in time in the same way as the upstream flow, while the vorticity remains constant. Thus the streamfunction spectra becomes a line spectra at frequency $\omega$, while the vorticity spectra tends to a delta function at zero frequency.

The observed frequency spectra of geostrophic streamfunction (not shown) and vorticity are basically different. Height spectra are characterized by very large contributions in the lower frequencies and a very sharp decrease with increasing frequency. Thus, it is difficult to evaluate the cause for the relatively minor changes in the contributions by different frequencies at different locations. These characteristics are partly due to the large degree of persistence that is observed in the height field. Rather than modify the observed data to filter out this effect we decided to use observed geostrophic vorticities to compare with model vorticities.

\section{b. Model results at selected grid points}

The model is better applied to a one-year sample since the results depend on the strength of the mean flow oscillation, which varies from year to year. For the following discussion we have selected the first winter as a test period. Fig. 6 depicts the time averages and standard deviations of vorticity at three levels.
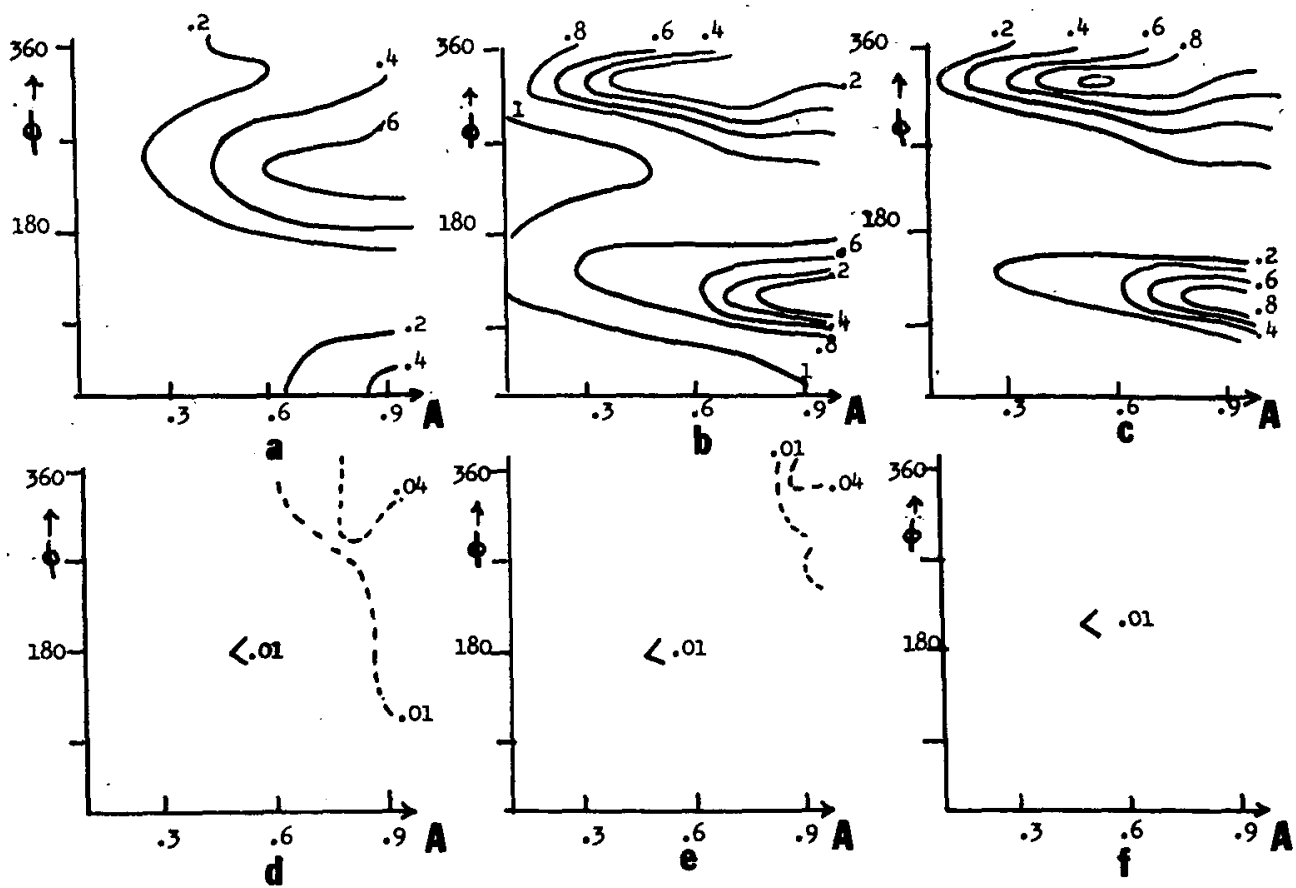

Fig. 4. Total variance of $g_{1}$ (a) and fractional contribution to the total variance of $g_{1}$ from frequency bands $\Delta f_{1}(\mathrm{~b}), \Delta f_{2}(\mathrm{c}), \Delta f_{3}$ (d), $\Delta f_{4}$ (e), and $\Delta f_{5}$ (f). 


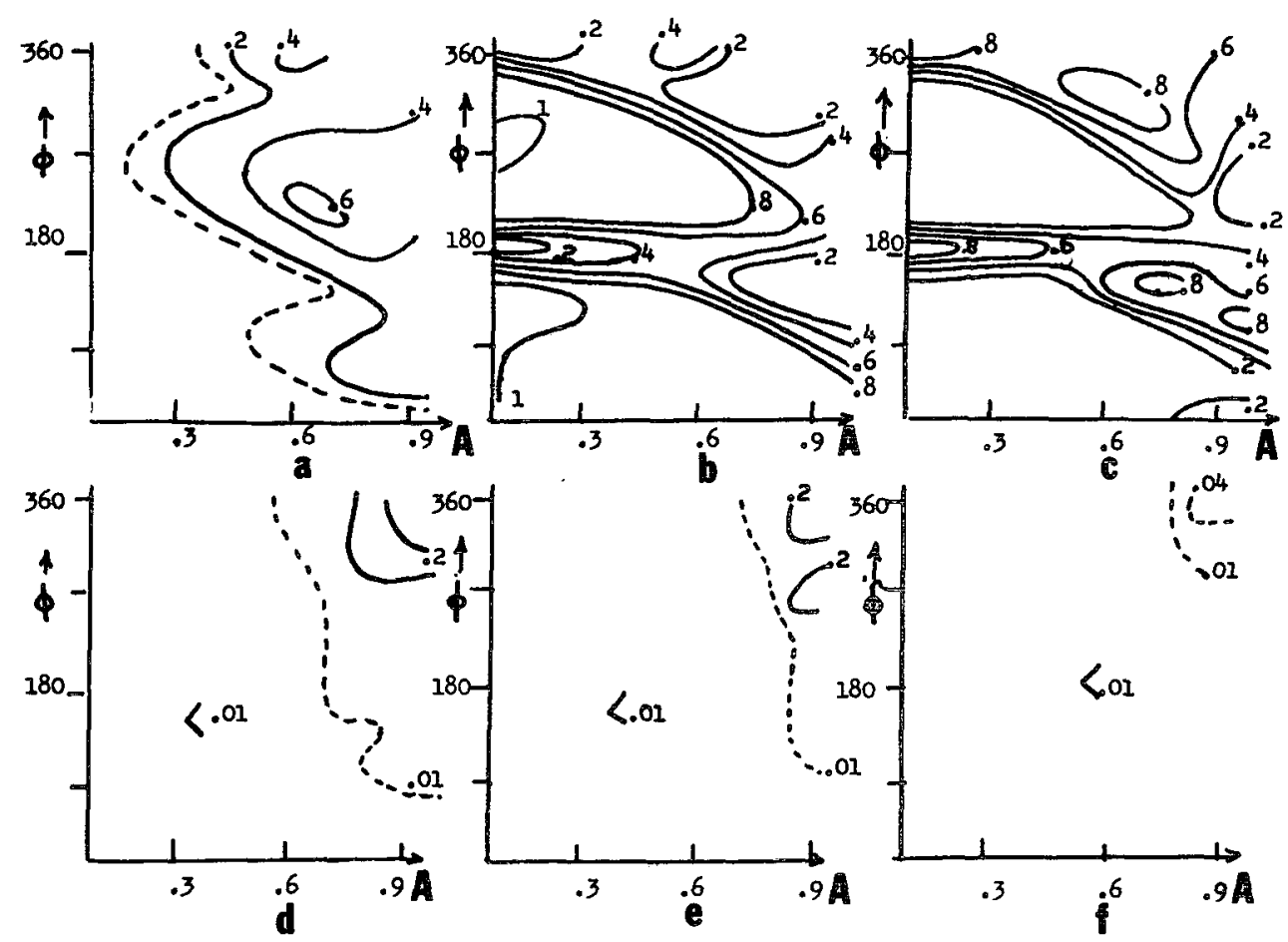

Frg. 5. As in Fig. 4 except for $g_{2}$.

The cell of maximum variability located over about $38^{\circ} \mathrm{N}$ and $102^{\circ} \mathrm{W}$ (western Oklahoma) is the one feature at $700 \mathrm{mb}$ which does not have a counterpart at higher levels. It is also located at the lee of the broadest sections of the Rockies and thus it seems reasonable to apply the mathematical orographic model at this latitude.

Observed vorticity spectra were compared with those obtained from the model at grid points $\mathrm{L}, \mathrm{M}, \mathrm{N}, \mathrm{O}$ (Fig. 1). These points were chosen about $10^{\circ}$ longitude apart to check the model at a variety of downstream distances. The respective model locations are $x=0.33$, 1.06, 1.7 and 2.5. The model was centered at $40^{\circ} \mathrm{N}$ and $700 \mathrm{mb}$ heights at grid points just off the central California coast' were chosen to obtain a time series of an upstream geostrophic zonal wind for the 1965-66 winter. Fig. 7 depicts its Fourier spectra. The values are non-dimensionalized by division with the stationary flow magnitude. The strong component at 32 days (98\% of the stationary flow value) justifies selection of $U_{A}(0)$ as $0.98 \sin (\omega \tau)+1.0$ where $\omega=(2 \pi / 32) \mathrm{day}^{-1}$. Other parameters were $C=0.8, B=1.93$, Ro $=0.096$ and $L=1.2$, which apply to the latitude of interest. A more general expression for $U_{A}(0)$ including higher frequencies would invalidate the assumption of a slowly oscillating upstream flow.

Fig. 8 shows the time variations of the streamfunction predicted by the model at points $\mathrm{L}, \mathrm{M}, \mathrm{N}, \mathrm{O}$. The intensity of the downstream flow and the wavelength of the induced wave depend upon the intensity of the upstream flow. The upstream flow attains maximum and minimum values at $\tau=\pi / 2 \omega$ and $\tau=3 \pi / 2 \omega$, respectively. As the flow decreases, the downstream wavelength and flow intensity decrease also. Thus, in the second half of the oscillation the selected locations are under the influence of ridge and trough positions which bring marked variations in the characteristics of the vorticity time series. The introduction of higher frequency components is easily detected in this figure from the rather smooth curve at point $\mathrm{L}$ to the larger oscillations at point $O$.

Fig. 9 is presented to further explain how these oscillations are induced. This figure depicts the streamfunction isopleth $\psi=0$ in a horizontal plane above the mountains at four instants within one oscillation of the mean flow. The intensity of the downstream flow can be inferred from the streamfunction values given in parentheses for grid points $\mathrm{L}$ and $\mathrm{M}$. The region $\alpha x<\pi / 2$ and $\alpha x>3 \pi / 2$ is characterized by anticyclonic vorticity, while the region $\pi / 2<\alpha x<3 \pi / 2$ has cyclonic vorticity. The time variations in Fig. 8 for point $L$ show no appreciable change in the value of the streamfunction while the upstream flow is strong, with a very small increase as the upstream flow decreases. From Fig. 9 it is easy to see that point $L$ is associated with anticyclonic vorticity at first, while it is later under the influence of the trough and cyclonic vorticity. At these later times the downstream flow is very weak and consequently this effect is hardly reflected in the streamfunction. Similar behavior is noted for point $M$. It is noteworthy that while $\mathrm{L}$ and $\mathrm{M}$ are almost within onefourth a wavelength for the first three times shown, 

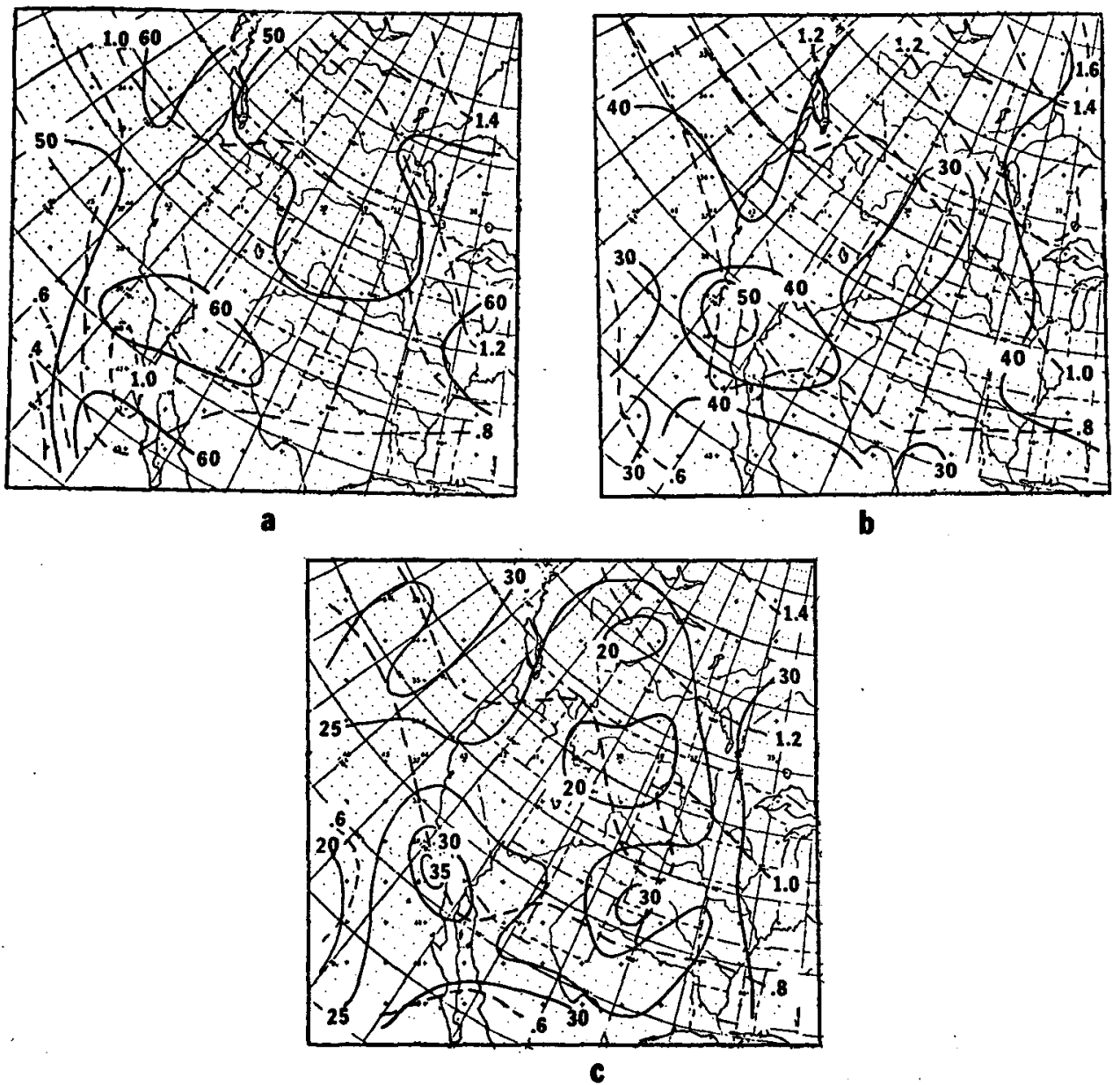

FIG. 6. Time average (dashed lines, in units of $10^{-4} \mathrm{~s}^{-1}$ ) and standard deviations (solid lines, in units of $10^{-6} \mathrm{~s}^{-1}$ ) for absolute geostrophic vorticities at 300,500 and $700 \mathrm{mb}$ (a, b and c, respectively) for the winter 1965-66.

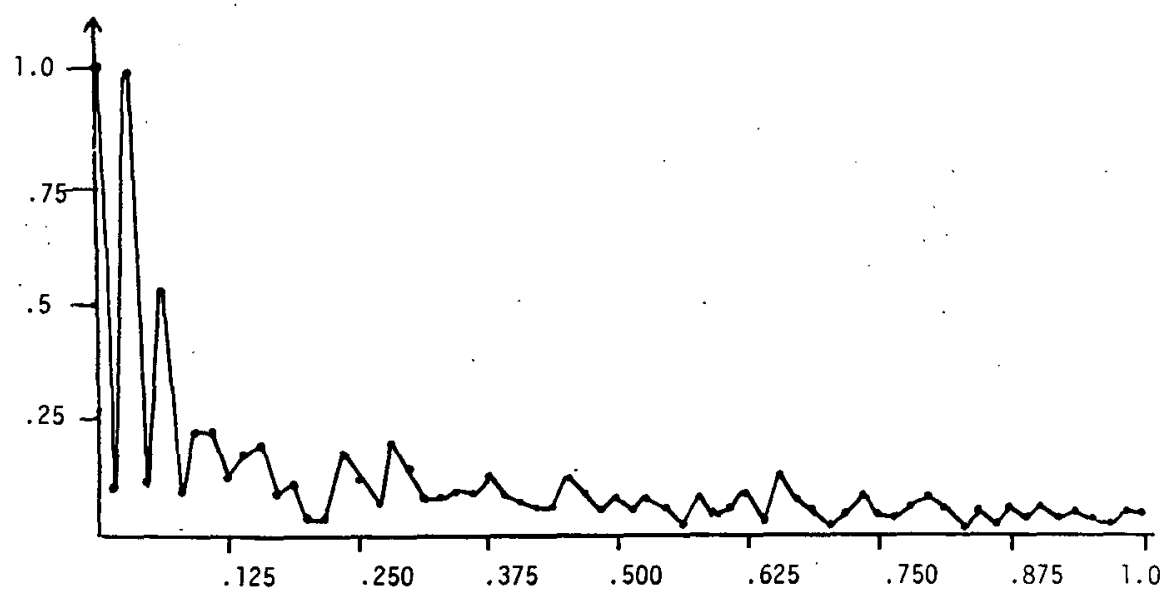

FIG. 7. Observed Fourier spectra of geostrophic zonal wind at $700 \mathrm{mb}$ in non-dimensional units versus cycles per day. 

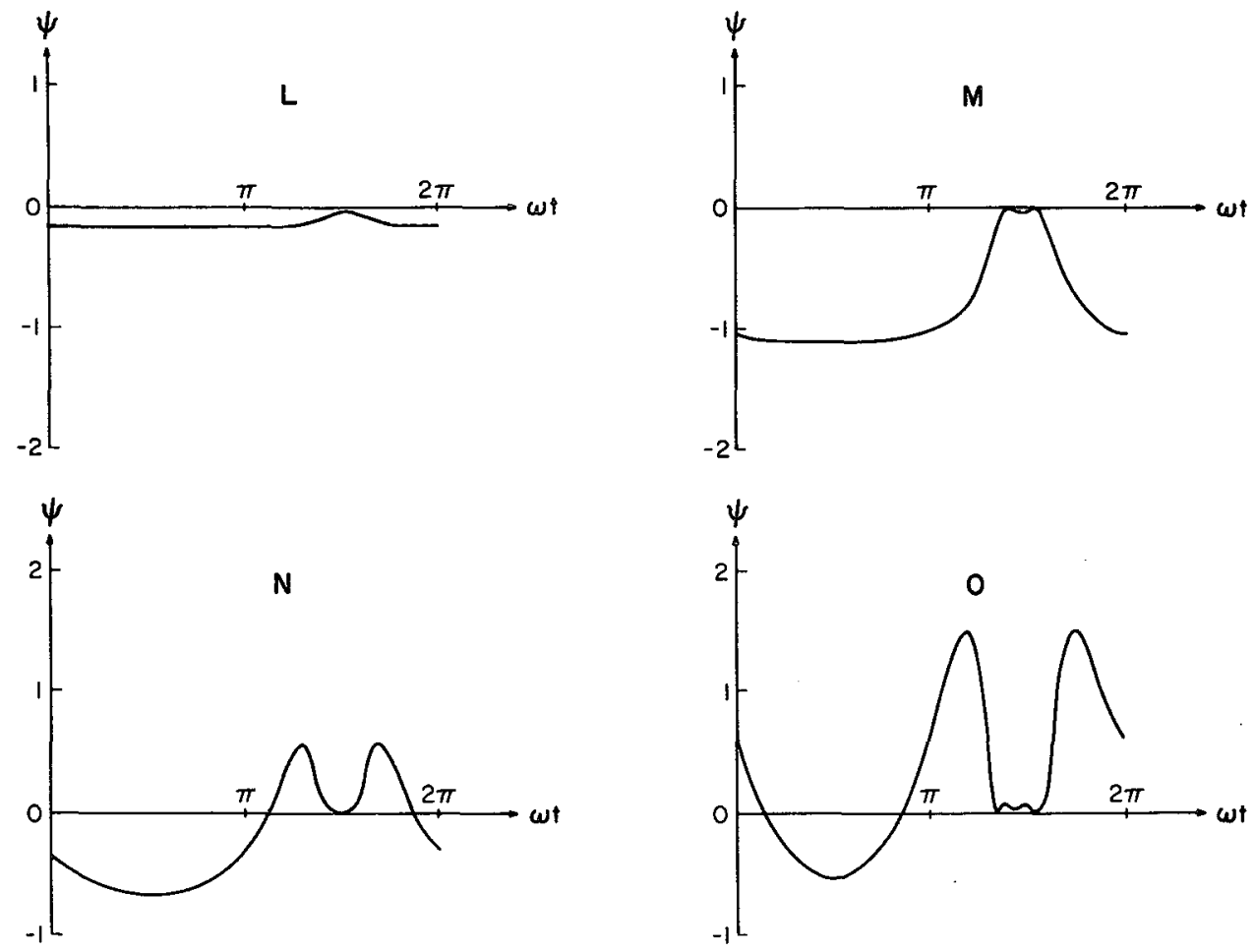

FIG. 8. Model streamfunction in non-dimensional units at grid points $L, M, M, O$.

they are about one wavelength apart when the flow is the weakest. Fig. 9 shows that the maximum deflection of the downstream flow is independent of the strength of the flow. This can be verified by examining
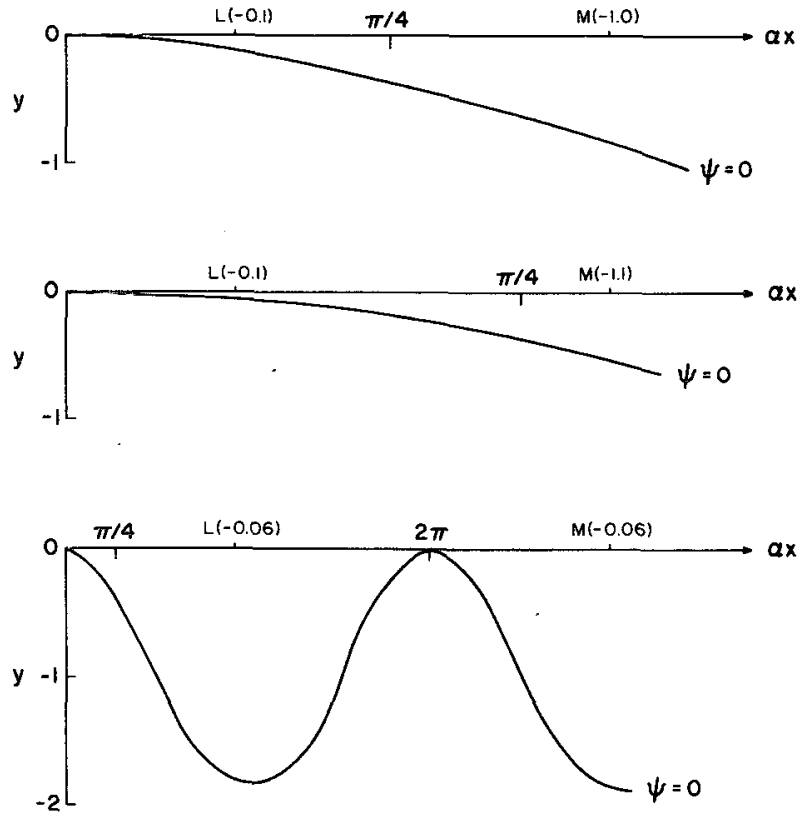

Fig. 9. Horizontal model configuration of $\psi=0$ at times $\tau=0$ and $\pi / \omega$ (upper diagram), $\tau=\pi / 2 \omega$ (middle diagram), and $\tau=3 \pi / 2 \omega$ (lower diagram) the $y$ position for the point $\alpha x=\pi / 4$. Similar interpretations hold for points $\mathrm{N}$ and $\mathrm{O}$.

Fig. 10 compares the vorticity spectra obtained from the model with those observed at $700 \mathrm{mb}$, in an attempt to isolate from the observed frequency spectra those characteristics which may result from the existing orography. The observed power spectra were transformed into a non-dimensional Fourier spectra for this purpose, using the same typical scales as for the model. Both the observed and predicted spectra show an eastward increase in the contribution of frequencies associated with 2-8 day periods. It is also of interest that the total time variability is predicted and observed to increase with longitude from over the ridge to the east

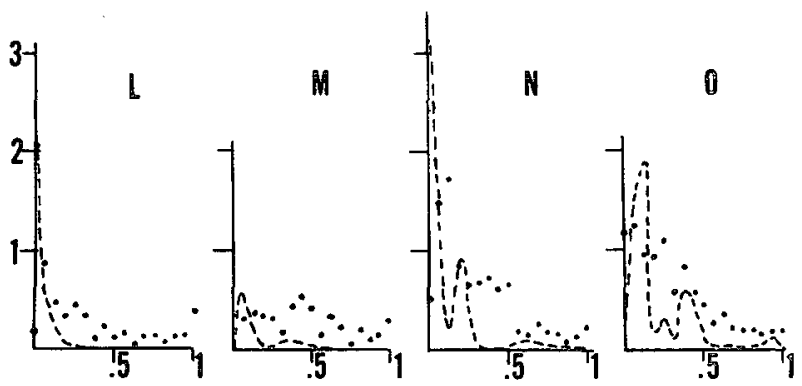

FIG. 10. Comparison of observed spectra (dots) for $700 \mathrm{mb}$ geostrophic vorticities at grid points $\mathrm{L}, \mathrm{M}, \mathrm{N}, \mathrm{O}$ for one-year sample with model spectra predictions (dashed lines), in nondimensional units, vs cycles per day. 
of the ridge in middle latitudes. Thus the proposed model may account for some of the observed features of the vorticity spectra, to a first approximation. A closer agreement is not expected due to the crudeness and limitations of the analytic model.

\section{Conclusions}

McClain (1960) has concluded from a case study of lee cyclogenesis that thermal influences are important to lee development. Baroclinic forecast experiments (Paegle and Kousky, 1972) on the development of the April 1965 Chicago tornado outbreak also suggest a substantial baroclinic contribution to the lee effect. The present study indicates that a very simple barotropic model of flow over a mountain is capable of simulating large cross-mountain increases of synoptic time scale (2-10 day) variance. These results are not necessarily contradictory, since it is difficult to isolate those aspects of development which are purely baroclinic from those which also have strong barotropic contributions in real data studies.

Major assumptions in the model described in Section 3 include necessity of separation of time scales, latitudinal invariance, and a very simple conservation law. Such assumptions may be desirable to isolate cause and effect in a first approximation and more general treatments would require numerical approaches.

Acknowledgments. We would like to thank M. G. Wurtele for his encouragement in the earliest phase of this research and one of the reviewers for a concise physical statement of the analytic model results.

The spectral computations were performed with the U.C.L.A. BMDX92 program modified for the University of Utah UNIVAC 1108 computer. The data were obtained from NOAA under Contract 1-35372.
This research was partially funded by NSF Grant ATM 73-00188.

\section{REFERENCES}

Batchelor, G. K., 1967: An Introduction to Fluid Dynamics. Cambridge University Press, $614 \mathrm{pp}$.

Bolin, B., 1950: On the influence of the earth's orography on the general character of the westerlies. Tellus, 2, 184-195.

Charney, J., and A. Eliassen, 1949: A numerical method for predicting the perturbation of the middle latitude westerlies. Tellus, 1, 38-54.

Kao, S. K., and L. L. Wendell, 1970: The kinetic energy of the large scale atmospheric motion in wavenumber-frequency space. I. Northern Hemisphere. J. Atmos. Sci., 27, 359-375.

- - R. L. Jenne and J. F. Sagendorf, 1970: The kinetic energy of large scale atmospheric motion in wavenumber-frequency space. II. Mid-troposphere of the Southern Hemisphere. J. Atmos. Sci., 27, 1008-1020.

- J. N. Paegle and W. E. Normington, 1974: Mountain effect on the motion in the atmosphere's boundary layer. Bound.-Layer Meteor., 7, 501-512.

Kasahara, A., and W. M. Washington, 1971 : General circulation experiments with a six-layer NCAR model, including orography, cloudiness and surface temperature calculations. $J$. Atmos. Sci., 28, 657-701.

Manabe, S., and T. B. Terpstra, 1974: The effects of mountains on the general circulation of the atmosphere as identified by numerical experiments. J. Atmos. Sci., 31, 3-42.

McClain, E. Paul, 1960: Some effects of the western cordillera of North America on cyclone activity. J. Meteor., 17, 104-115.

Mintz, Y., 1965 : Very long-term global integration of the primitive equations of atmospheric motions. WMO Tech. Note No. 66, 141-167.

Paegle, J., and V. Kousky, 1972 : Lectures on Synoptic Meteorology. University of Utah.

Paegle, J. N., and L. P. Keirulff, 1974: Synoptic climatology of $500 \mathrm{mb}$ winter types. $J$. Appl. Meteor., 13, 205-212.

- - and J. Paegle, 1975: On the observed characteristics of quasi-geostrophic turbulence. Mon. Wea. Rev., 103, 10551062.

Queney, P., 1948: The problem of air flow over mountains: A summary of theoretical studies. Bull. Amer. Meteor. Soc., 29, 16-26. 